\title{
ON A CLASS OF GENERAL LINEAR GROUP WHOSE DETERMINANT IS THE SAME AS THE TRACE
}

\author{
U.E. Edeke ${ }^{1 \S}$, O.E. Ntekim ${ }^{2}$, E.I. Enang ${ }^{3}$ \\ ${ }^{1,2}$ Department of Mathematics \\ University of Calabar \\ NIGERIA \\ ${ }^{3}$ Department of Statistics \\ University of Calabar \\ NIGERIA
}

\begin{abstract}
General Linear Groups are examples of Topological Groups. In this work, a class of $G L(2, \mathbb{R})$ whose trace and determinant are equal is constructed. The proposed $G L(2, \mathbb{R})$ class is further endowed with Euclidean topology and shown to be a topological group. A sequence of the constructed $G L(n, \mathbb{R})$ and its generalization are also presented.
\end{abstract}

AMS Subject Classification: 22A05, 22A10, 57P02

Key Words: topological group, general linear group, Euclidean topology, trace, determinant

\section{Introduction}

General Linear Group $G L(n, \mathbb{R})$ is a group of invertible square matrices of order $n$ and the elements are from the set of real numbers $\mathbb{R}$. This group is not compact because matrices with positive and negative determinant split it into two groups. That is $G L(n, \mathbb{R})=G L^{+}(n, \mathbb{R}) \cup G L^{-}(n, \mathbb{R})$, where $G L^{+}(n, \mathbb{R})$ is a group of invertible square matrices with positive determinant and $G L^{-}(n, \mathbb{R})$

Received: November 10, 2015

Published: February 15, 2016

$\S$ Correspondence author (c) 2016 Academic Publications, Ltd.

url: www.acadpubl.eu 
with negative determinant, they are subsets of the matrix algebra $M(n, \mathbb{R})$. When a Hausdorff topology is defined on this group such that the operations of multiplication and inverse are continuous, the group becomes a topological group, see[1] and [7]. The topology of $G L(n, \mathbb{R})$ is an induced topology from $M(n, \mathbb{R}),[1]$. Let $A=\left(a_{i j}\right)$ and $B=\left(b_{i j}\right)$ be two matrices and let $d(A, B)=\max _{i, j}\left|a_{i, j}-b_{i, j}\right|$ be the distance between $\mathrm{A}$ and $\mathrm{B}$. This defines a metric on the space $M(n, \mathbb{R})$ and its topology is defined by considering its spherical neighborhood as its basis elements. Since $G L(n, \mathbb{R}) \subset M(n, \mathbb{R})$ it therefore carries an induced topology from $M(n, \mathbb{R})$.

In this paper, a special kind of $G L(n, \mathbb{R})$ whose determinant and trace are the same is constructed and is further showed that it is a topological group when endowed with an induced topology from $M(n, \mathbb{R})$. Multiplication is not necessarily associative in this group, for $A, B \in \mathrm{GL}(\mathrm{n}, \mathrm{R}),[A, B]=A B-B A$. This defines bilinear multiplication on $G L(n, \mathbb{R})$ hence, it is also a Lie algebra, [6]

\section{Preliminaries}

In this section, preliminaries concerning topological groups, Lie groups, Lie algebras and differentiable manifold are presented.

2.1 Topological Group : Let $G$ be a group (considered multiplicatively) on which a (Hausdorff) topology is defined such that the maps

(i) $\left(g_{1}, g_{2}\right) \longmapsto g_{1} g_{2}: G \times G \rightarrow G$.

(ii) $g \longmapsto g^{-1}: G \times G$ are continuous, then $G$ is called a topological group. In a nutshell, a group $G$ endowed with a Hausdorff topology such that the operations of multiplication and inverse are continuous is called a topological group [2]. Examples of topological group are the additive group $\mathbb{R}:=(\mathbb{R},+,||$. of real numbers equipped with the modulus topology, the General Linear group $G L(n, R)$, any abstract group $\mathrm{G}$ with the discrete topology assigned to it etc.

According to [1],continuity of multiplication is equivalent to the condition that for $g_{1} g_{2}=g_{3}$ and for any neighborhood $V_{3}$ of $g_{3}$ there exist neighborhoods $V_{1}$ of $g_{1}$ and $V_{2}$ of $g_{2}$ such that $V_{1} V_{2} \subset V_{3}$ where

$$
V_{1} V_{2}=\left\{x y \mid x \in V_{1}, y \in V_{2}\right\} .
$$

Also, continuity of taking inverse is equivalent to the condition that for any neighborhood $V$ of $g^{-1}$ there exist a neighborhood $U$ of $g$ such that $U^{-1} \subset V$, where 


$$
U^{-1}=\left\{x^{-1} \mid x \in U\right\}
$$

2.2 Chart: Let $M$ be a topological space that satisfies the Hausdorff separation axiom. An open chart on $\mathrm{M}$ is a pair $(U, \varphi)$ where $\mathrm{U}$ is an open subset of $\mathrm{M}$ and $\varphi$ is a homeomorphism of $\mathrm{U}$ onto an open subset of $\mathbb{R}^{m}$ [5].

2.3 Differentiable Structure:A differentiable structure of dimension $m$ on a Hausdorff topological space $M$ is defined as a collection of open charts $\left(U_{\alpha}, \varphi_{\alpha}\right)_{\alpha \in A}$ on $M$ where $\varphi_{\alpha}\left(U_{\alpha}\right)$ is an open subset of $\mathbb{R}^{m}$ with the following conditions:

(i) $M=\cup_{\alpha \in A} U_{\alpha}$;

(ii) For each pair $\alpha, \beta \in A$. The composition map $\varphi_{\beta} \circ \varphi_{\alpha}^{-1}$ is a differentiable mapping of $\varphi_{\alpha}\left(U_{\alpha} \cap U_{\beta}\right)$ onto $\varphi_{\beta}\left(U_{\alpha} \cap U_{\beta}\right)$;

(iii) The collection $\left(U_{\alpha}, \varphi_{\alpha}\right)_{\alpha \in A}$ is a maximal family of open charts for which conditions (i)\& (ii) hold [5].

2.4 Differentiable Manifold: A differentiable manifold or a $C^{\infty}$ - manifold of dimension $\mathrm{m}$ is a Hausdorff space endowed with a diffentiable structure of dimension $\mathrm{m}$.

Definitions 2.5 to 2.7 are from [4], [2] and [6].

2.5 Algebra: An algebra over a field $\mathbb{I}(\mathbb{C}$ or $\mathbb{R})$ is a linear space A over $\mathbb{F}$ together with a mapping $(x, y) \mapsto x y$ of $\mathrm{A} \times \mathrm{A}$ into $\mathrm{A}$ that satisfies the following axioms:

(i) $x(y z)=(x y) z$;

(ii) $x(y+z)=x y+x z,(x+y) z=x z+y z$;

(iii) $(\alpha x) y=\alpha(x y)=x(\alpha y)$.

The above algebra is called linear associative algebra, the field $\mathbb{F}$ is called the scalar field of $\mathrm{A}$. If $\mathbb{F}=\mathbb{R}, \mathrm{A}$ is called a real algebra, and if $\mathbb{F}=\mathbb{C}, \mathrm{A}$ is called a complex algebra, The mapping $(x, y) \longmapsto x y$ is called a bilinear vector product.

2.6 Lie Algebra: Let $\mathbb{K}$ be a field of characteristic zero(0) and let $\mathfrak{g}$ be a vector space over $\mathbb{K} . \mathfrak{g}$ is said to be a Lie algebra if and only if there is a bilinear multiplication

$$
[., .]:=(x, y) \longmapsto[x, y]: g \times \mathfrak{g} \longrightarrow \mathfrak{g} \text { on } \mathfrak{g}
$$

such that:

(i) $[x, x]=0$; 
(ii) $[x[y, z]]+[y,[z, x]]+[z[x, y]]=0 . \forall x, y, z \in \mathfrak{g}$.Property (ii) above is called the Jacobi identity.

The bilinearity of the map [.,.] means that:

(a) $[a x+b y, z]=a[x, z]+b[y, z]$;

(b) $[x, b y+c z]=b[x, y]+[x, z] . \forall a, b, c \in \mathbb{K}$ and $\forall x, y, z \in g$.

The bilinear multiplication which is the Lie bracket is not necessarily associative. A lie algebra $\mathfrak{g}$ is said to be finite dimensional if the underlying vector space $\mathfrak{g}$ is finite dimensional, otherwise the Lie algebra $\mathfrak{g}$ is infinite dimensional. Lie algebras arise in nature as vector space of linear transformations endowed with a new operation which is in general neither commutative nor associative. A vector subspace $k$ of a Lie algebra $\mathfrak{g}$ is called a subalgebra of $\mathfrak{g}$ if

$$
[k, k] \subseteq k
$$

In this case $k$ is a Lie algebra under the operation induced from $g$. A subalgebra $k$ of a Lie algebra $\mathfrak{g}$ is said to be an ideal if $[\mathfrak{g}, k] \subseteq k$. A Lie algebra $\mathfrak{g}$ is simple if its only ideal are 0 and $\mathfrak{g}$. A Lie algebra $\mathfrak{g}$ is said to be Abelian (= commutative) if $[x, y]=[y, x], \forall x, y \in \mathfrak{g}$.

2.7 Lie Groups : A Lie group $\mathrm{G}$ is a set that is a $C^{\infty}$ - manifold and a topological group such that the manifold and the group structures on $\mathrm{G}$ are compatible in the sense that the maps $\left(g_{1}, g_{2}\right) \longmapsto g_{1} g_{2}: G \times G \longrightarrow G$ and $g$ $\longmapsto g^{-1}: G \longrightarrow G$ are $C^{\infty}$

\section{Main Result}

A kind of $G L(n, \mathbb{R})$ where the determinant is equal to its trace is constructed. As earlier defined, $G L(n, \mathbb{R})$ is a subset of the algebra $M(n, \mathbb{R})$ whose determinant is different from zero. In other words, it is a group of invertible matrices.

We construct a sequence below and study some of its properties.

$$
M_{n}=\left\{A_{n} \in G L(n, \mathbb{R}) \mid \operatorname{det}\left(A_{n}\right)=\operatorname{tra}\left(A_{n}\right)\right\}
$$

. Consider some of the terms of the sequence of matrix $M_{n} \cdot M_{1}=\left(\begin{array}{cc}0 & 1 \\ -1 & 1\end{array}\right)$, $M_{2}=\left(\begin{array}{cc}0 & 2 \\ -2 & 4\end{array}\right), M_{3}=\left(\begin{array}{cc}0 & 3 \\ -3 & 9\end{array}\right), \ldots, M_{n-1}=\left(\begin{array}{cc}0 & n-1 \\ 1-n & (n-1)^{2}\end{array}\right)$, $M_{n}=\left(\begin{array}{cc}0 & n \\ -n & n^{2}\end{array}\right)$. Let $M_{n}\left(a_{2,2}\right)$ be the element in row two and column two 
of the $n^{t h}$ sequence of the matrix $M$. Let the sequence below be the ascending element of few members of $M_{n}\left(a_{2,2}\right)$ listed above

$$
1 \longrightarrow 4 \longrightarrow 9 \longrightarrow 16 \longrightarrow 25 \longrightarrow \ldots
$$

Observe that the difference between the first term and the second term is 3 , second and third is 5, third and fourth is 7, fourth and fifth is 9 . Therefore, the general term for generating elements of $M_{n}\left(a_{2,2}\right)$ is presented below

$$
M_{n}\left(a_{2,2}\right)=2\left[M_{n-1}\left(a_{2,2}\right)+1\right]-M_{n-2}\left(a_{2,2}\right),
$$

for $n \geq 3$.

We claim that this sequence of matrix whose $n^{\text {th }}$ term is given above is a topological group. The sketch of the proof is presented as follows. We restrict our investigation to only square matrices of order two that the trace and determinant are the same. Let $A=\left(a_{i, j}\right)$ represents a typical element in $M_{2}(\mathbb{R})$. We identify $M_{2}(\mathbb{R})$ with the Euclidean space $\mathbb{R}^{4}$ by associating $A=\left(a_{i, j}\right)$ with the points $\left(a_{11}, a_{12}, a_{21}, a_{22}\right)$. The identification gives us a topology on $M_{2}(\mathbb{R})$ which is the topology of $\mathbb{R}$. Next we show that the matrix multiplication

$$
m: M_{2}(\mathbb{R}) \times M_{2}(\mathbb{R}) \rightarrow M_{2}(\mathbb{R})
$$

is continuous. To this end, we examine the formula for the entries of the product matrix. If $A=\left(a_{i j}\right)$ and $B=\left(b_{i j}\right)$, then $m(A, B)=A B$. The $(i, j)^{t h}$ entry of AB is therefore $\sum_{k=1}^{n} a_{i k} b_{k j} . M_{2}(\mathbb{R})$ has the topology of the product space $\mathbb{R}^{4}$ and for each i,j satisfying $1 \leq i, j \leq n$, we have $\Pi_{i j}:=A \mapsto a_{i j}: M_{2}(\mathbb{R}) \rightarrow \mathbb{R}$ which sends a given matrix $A$ to its $(i, j)^{\text {th }}$ entry. The multiplication map $m$ is continuous if and only if the composite function

$$
\begin{gathered}
m: M_{2}(\mathbb{R}) \times M_{2}(\mathbb{R}) \rightarrow M_{2}(\mathbb{R}) \\
\Pi_{i j}: M_{2}(\mathbb{R}) \rightarrow \mathbb{R} \\
\Pi_{i j} \circ m: M_{2}(\mathbb{R}) \times M_{2}(\mathbb{R}) \rightarrow \mathbb{R}
\end{gathered}
$$

are continuous. But $\Pi_{i j} \circ m(A, B)=\sum_{k=1}^{n} a_{i k} b_{k j}$ is a polynomial in the entries of $\mathrm{A}$ and B. Therefore, $\Pi_{i j} \circ m$ is continuous, thus $m$ is continuous.

The elements of $G L(2, \mathbb{R})$ are invertible elements of $M_{2}(\mathbb{R})$. If we endow $G L(2, \mathbb{R})$ the subspace topology from $M_{2}(\mathbb{R})$, then the multiplication

$$
m: G L(2, \mathbb{R}) \times G L(2, \mathbb{R}) \rightarrow G L(2, \mathbb{R})
$$

is continuous. What remains is to show that the inverse map 


$$
i: G L(2, \mathbb{R}) \rightarrow G L(2, \mathbb{R}) \subseteq \mathbb{R}^{4}
$$

is continuous if and only if all composite functions

$$
\begin{gathered}
i: G L(2, \mathbb{R}) \rightarrow G L(2, \mathbb{R}) \\
\Pi_{j k}: G L(2, \mathbb{R}) \rightarrow \mathbb{R} \\
\Pi_{j k} \circ i: G L(2, \mathbb{R}) \rightarrow \mathbb{R}
\end{gathered}
$$

are continuous for $1 \leq j, k \leq n$. The map $\Pi_{j k} \circ i$ sends a matrix $A$ to the $(j, k)^{t h}$ element of $A^{-1}$. Notice that the determinant of $A$ and the cofactor of $A$ are polynomials in the entries of $A$ and the determinant of $A$ does not vanish on $G L(2, \mathbb{R})$ and our composite map $\Pi_{j k} \circ i$ is continuous. This completes the proof that the group of matrices that the trace is equal to the determinant is a topological group.

\section{References}

[1] G. Bachman, Elements of Abstract Harmonic Analysis, Academic Press, New York (1964).

[2] U.N. Bassey, Lecture Notes on Differentiable Manifolds, Lie Groups and Lie Algebras, University of Ibadan( 2013).

[3] D. Beltita and M. Nicolae, On Universal Enveloping Algebra in a Topological Setting, arXiv: 1402.0186v2 [math. FA] 25 Feb 2014.

[4] J. Duncan and F.F. Bonsall, Complete Normed Algebras, Berlin Heidelberg, New York (1973).

[5] S. Helgason, Differential Geometry and Symmetric Spaces, Academic Press, New York and London (1962).

[6] V.S. Varadarajan, Lie Groups, Lie Algebras, and Their Representations, Springer - Verlag New york Berlin Heidelberg Tokyo( 1984).

[7] J.A. Wolf, Harmonic Analysis on Commutative Spaces, American Mathematical Society, (2007). 